\title{
Sustainability and the robust delivery of resources
}

\author{
A. Kharrazi, M. Yarime \& G. Trencher \\ Graduate Program in Sustainability Science, \\ Graduate School of Frontier Sciences, University of Tokyo, Japan
}

\begin{abstract}
Sustainability as a concept has multiple disparate perspectives stemming from different related disciplines which either maintain ambiguous interpretations or concentrate on metrics pertaining to single aspects of a system. An ecological information-based approach is a holistic measurement which examines the robustness of flows as an important aspect of sustainability. This paper evaluates this approach by applying it to four economic resource trade flow networks. From the perspective of biomimicry, it appears that these networks can achieve higher levels of efficiency without weakening their robustness to resource delivery.

Keywords: economic resource networks, ecological information theory, indicators, robustness, sustainability.
\end{abstract}

\section{Introduction}

Sustainability is an increasingly important new paradigm of the modern age upon which to base future policies. However, a universally accepted definition for sustainability has been difficult to achieve as the terminology remains open to different interpretations. Quantification of sustainability could create a common ground to compare alternative policies. However, existing single issue indicators examine only partial dimensions of a system. The challenge lies in the measurement of sustainability as a holistic metric at the system level. In this light, the ecological information-based approach is a promising holistic metric for examining levels of robustness which is a critical determinant of the sustainability of a system. 
Being robust to changes in the flows within a network, resulting for example from stresses such as extinction, drought, climate change, and recession, is considered important for a system to be sustainable [1]. However, there is a trade-off between a system's efficiency and redundancy; high levels of efficiency tend to weaken the system's ability to respond to shocks or disruptions to its internal flows, while high levels of redundancy are costly to maintain and tend to weaken the system's internal development and its ability to distribute flows in an orderly way. An ecological information-based approach measures the system's robustness, as a balance between its efficiency and redundancy, as an intensive parameter. Therefore, as a result of both the intensive and extensive parameters, the ecological information-based approach can potentially be beneficial towards the aim of quantifying sustainability. It is necessary to note that the ecological information-based approach utilizes macro level measurements of the patterns that arise from the flow structure of the system under study. While these macro level measurements do not directly measure socio-environmental demands and or pressures, these issues are implicitly reflected in the flow structure of the system.

While this approach has been applied to natural systems there have been few studies to apply it to human-environmental systems [2]. The main contributions of this paper are to further advance this method by demonstrating what can biomimicry as a normative paradigm tell us about the system-level configurations of the networks. Specifically for the discussion of this paper, oil, world commodity, OECD-BRIC commodity, and OECD-BRIC foreign direct investment trade flow networks were examined. We chose these networks because they are human networks having high volumes of trade at the global scale and which are directly related to the availability of natural resources.

\section{Ecological information-based approach}

The ecological information-based approach uses the Shannon Index (SI), where the ratio between the presence and absence of information, i.e., certainty and uncertainty or order and non-order, plays a central role in determining the longterm robustness of a system. Claude Shannon was concerned with the degree of uncertainty or surprise associated with predicting words in a string of text. His underlying assumption was that the difficulty in predicting which word will appear after the other in a string of text increases with the diversity of the vocabulary and the evenness of the probability of each word occurring in the text. The Shannon Index is a measure of the entropy (uncertainty or degree of surprise) associated with such a prediction. For a networked system the SI is redefined as [3]:

$$
H=-k \sum_{i, j} \frac{T_{i j}}{T_{. .}} \log \frac{T_{i j}}{T_{. .}}
$$




$$
\begin{gathered}
X=k \sum_{i, j} \frac{T_{i j}}{T_{. .}} \log \frac{T_{i j} T_{. .}}{T_{i .} T_{. j}} \\
\psi=-k \sum_{i, j} \frac{T_{i j}}{T_{. .}} \log \frac{T_{i j}^{2}}{T_{i .} T_{. j}}
\end{gathered}
$$

In information theory, variable $X$, known as the "average mutual information" $(A M I)$, is the average measure of degrees of constraints in the network for all flows $T_{i j}$. The variable $X$ therefore, can be interpreted as the difference between uncertainty of the destination of the flow leaving node $i$ and the uncertainty that remains after the flow from node $i$ to node $j$ is observed. Variable $\psi$, known as the conditional entropy, is the measure of the average degrees of freedom in the network for all flows $T_{i j}$. Given the degree of constraints of a flow leaving node $i, \psi$ can be interpreted as the remaining choice of pathways for flows going to node $j$. Both of these metrics, $\psi$ and $X$, are dimensionless and based on units of information depending on the base logarithm used in their calculation, e.g. bits if the base 2 logarithm is used or nats if the natural logarithm is used.

Usually, TST, which is a measure of the size of the system, is used as the constant $k$. This leads to three new terms. The scaled aggregate system level indeterminacy $(C)$, describes the system's "capacity" for growth and development:

$$
C=T_{. .} . H
$$

The system's capacity, which is always positive, is the upper limit of the total system indeterminacy, or in ecology terms, the maximum potential of a system to evolve or to self-organize. This in turn can be defined as $C=A+\phi$. A network with a higher $(C)$ can be considered to have more potential for changing or evolving its structure. The scaled average mutual constraint of the system $(A)$ is called the system "ascendency" or system efficiency. Ascendency describes the degree to which a system efficiently distributes flows among its nodes, and therefore can be viewed as the structural backbone maintaining the integrity of the system's efficient configuration of flows. In ecology, systems having ascendency levels that are higher than overhead levels, described next, are viewed as more evolved systems that have survived the disturbances arising from their environment and have overtime solidified particular specializations in their network configuration of flows. Ascendency is defined as:

$$
A=T_{. .} . X
$$

The scaled conditional entropy $(\phi)$ is called the system "overhead" or system redundancy [4]. Overhead $(\phi)$, describes the flexibility that a system maintains for overcoming disruptions and shocks to its network. After the flows of a system are subjected to some form of stress that system may exhibit lower levels 
of overhead; this may be indicative that a portion of the overhead has been depleted. Overhead is defined as:

$$
\phi=T_{. .} \cdot \psi
$$

To help determine where such a balance between constraint in the system and flexibility in the system might lie, the relative order in the system is introduced as: $\alpha=A / C$, where $0 \leq \alpha \leq 1$. While keeping in mind that order itself is a result of the opposing tendencies within the system, the ratio $\alpha$, is a convenient way to express the degree of which order, as defined through the metric ascendency, dominates the system. Taking the measure of relative order and multiplying it by the Boltzmann measure of its disorder robustness can be derived as $R$, which depends on both order and lack of order in a system [5] and is expressed as:

$$
R=-\alpha \log (\alpha)
$$

As globalization increases, more economic resources are shared across borders. Therefore, in addition to the limited availability of these resources, the robust delivery of these resources is a challenge that needs to be addressed. Intensive dimensions of a flow network are derived from the structure of the network and how the network distributes a resource. Therefore, to attain better insight into how flows are distributed within a network and to also define a measurement of the robustness of the network at the system level the anatomy of the network must be characterized. Like ecological networks, economic resource networks need efficient distribution and the flexibility to adapt to system-level stresses, such as droughts, recession, and natural calamities. Using the application of the ecological information-based approach, we can derive a measurement for the robustness of these networks. While the ecological information-based approach can in principle be applied to any economic resource network, including urban, regional, national, and international levels, in reality, research is limited by data availability.

\section{Data}

We have applied the ecological information-based approach to four economic resource trade flow networks: 1) oil, 2) world commodity, 3) OECD commodity, and 4) OECD foreign direct investment. We chose these networks because they represent economic resource networks having high volumes of trade at the global scale and because they are directly related to environmental issues such as the availability of natural resources.

\section{Results}

The four economic resource trade flow networks examined in this paper indicated that these networks maintain on average low degrees of order $(\alpha)$, that is, higher levels of redundancy in their configurations. In comparison among the four networks, oil trade maintained the highest levels of robustness $(R)$. The trade networks for global and OECD-BRIC commodity respectively maintained 
on average the lowest levels of robustness $(R)$. The findings for all networks are summarized in Table 1.

Table 1: The average degrees of order $(\alpha)$ and corresponding levels of robustness $(R)$ for four economic resource trade flow networks.

\begin{tabular}{lcc}
\hline Economic Resource Trade Network & $(\alpha)$ & $(R)$ \\
Oil (2007-2011) & 0.199 & 0.321 \\
Global Commodity (1962-2010) & 0.092 & 0.222 \\
OECD-BRIC Commodity (1988-2010) & 0.086 & 0.211 \\
OECD-BRIC FDI (1985-2009) & 0.129 & 0.263 \\
\hline
\end{tabular}

\section{Discussion}

Due to higher levels of redundancy in their network pathways, the humanenvironmental networks examined in this study are less robust than natural networks [2] examined in previous studies. This was counterintuitive because one would expect human-designed networks to be more efficient. One reason might be natural evolution that has over a long time optimized the robustness of natural systems by trial and error. If this is indeed the case, then the networks we have examined might become more robust if further autocatalysis could be achieved and redundant pathways replaced with more efficient pathways.

However, not all human influenced networks have been found to maintain higher levels of redundancy. For example, previous research in urban water systems indicates higher levels of organization, with efficiency encompassing more than half of the developmental capacity of the system [5]. In fact, these systems were found to be over-efficient in regard to the network's robustness, making them inflexible or brittle. Here, one could argue that urban water systems are designed and established more explicitly through human intervention. Whereas, the economic resources networks examined are rather a spontaneous structure emerging from numerous interactions and less likely to be controlled by a single entity. Another factor in this disparity can be attributed to data availability. Specifically, economic networks and their flows are tracked and recorded with higher levels of details. Therefore, we cannot make a general statement about the level of organization in human-environmental networks at this point. More human-environmental networks, at different levels of granularity, need to be examined.

Configurations resulting in more robust systems can be seen as fulfilling the requirements of the intensive dimension of sustainability. Therefore, in combination with requirements of the extensive dimensions, such as reduction of total resource used, measurement of intensive dimensions can be viewed as a beneficial direction to pursue for policy making. For policy makers, the ecological information-based approach can be useful in evaluating the effect of a change in the flow configuration of the network. However given that this is a 
system-level approach based on measured flows between compartments, the underlying socio-economic dynamics defining these flows is not explicitly considered. It is beyond the scope of this paper to suggest new alterations to the configuration of the network. A future challenge will be to link the patterns observed by the ecological information-based approach to the socio-economic dynamics underlying the flows within the studied network.

Mimicking the way that nature organizes its flow configuration is not an easy task. Natural systems are shaped by evolutionary dynamics which are forceful, cruel, and brutally effective. Achieving similar dynamism in a human socioeconomic system may not be possible or desirable. Nevertheless, assuming that the mimicry of nature is beneficial in the organization of flow configurations, what could be achieved if the human-environmental networks that we have examined were made more efficient to achieve higher levels of robustness? We suggest that the benefit is more robust delivery of economic resources, achieved by balancing efficient delivery of resources with the ability of the system to withstand perturbations to its network structure.

\section{Conclusions}

While the ecological information-based approach can help to address the intensive dimensions of sustainability, such as efficiency, redundancy, and robustness, the approach is weak in addressing extensive dimensions of sustainability, specifically those concerning the need to quantify the total consumption and availability of resources. Although the total system throughput in principle reflects the total resources used in the system, this metric does not calculate the availability of resources. Furthermore, the metrics of ascendency and overhead, the scaled derivatives of efficiency and redundancy with respect to the total system throughput, are difficult to intuitively grasp because numerically they are always greater than the size of the network based on the total resources consumed. Finally, while this approach measures the robustness of a system, the challenge here is to find the optimal level of robustness in a system. One way of achieving this is to accept the biomimicry of nature as a normative paradigm. The other way would be a data-intensive approach where a range of possible network configurations could be mapped.

\section{References}

[1] Holling C, Gunderson L (2002) Resilience and adaptive cycles. In: Holling, C., Gunderson, L. (Eds.), Panarchy: Understanding Transformations in Human and Natural Systems. Island Press, Washington D.C.

[2] Goerner S, Lietaer B, Ulanowicz RE (2009) Quantifying economic sustainability: Implications for free-enterprise theory, policy and practice. Ecological Economics 69(1): 76-81.

[3] Ulanowicz RE, Norden JS (1990) Symmetrical overhead in flow networks. International Journal of Systems Science 21:429-437. 
[4] Ulanowicz RE (2001) Information theory in ecology. Computers \& Chemistry 25: 393-9.

[5] Li Y, Yang ZF (2011) Quantifying the sustainability of water use systems: calculating the balance between network efficiency and resilience. Ecological Modelling 222, 1771-1780. 